\title{
TEMPERATURE-STABLE HIGH-Q ALN-ON-SILICON RESONATORS WITH EMBEDDED ARRAY OF OXIDE PILLARS
}

\author{
R. Tabrizian, G. Casinovi, and F. Ayazi \\ Georgia Institue of Technology, Atlanta, Georgia, USA
}

\begin{abstract}
This paper reports on a new temperature compensation technique for high- $Q$ AlN-on-Silicon bulk acoustic wave resonators. A uniform array of silicon dioxide $\left(\mathrm{SiO}_{2}\right)$ pillars are formed in the silicon body of the resonator to generate a composite resonator with a near-zero temperature coefficient of frequency (TCF). At a resonance frequency of $24 \mathrm{MHz}$, a total frequency drift of $90 \mathrm{ppm}$ over the temperature range of $-20{ }^{\circ} \mathrm{C}$ to $100{ }^{\circ} \mathrm{C}$ was measured while $Q$ exceeded 10,000 at all temperatures. This compensation technique is applicable to bulk acoustic resonators with thick silicon substrate that demonstrate high $Q$ as well as good power handling and linearity.
\end{abstract}

\section{INTRODUCTION}

AlN-on-Silicon resonators are characterized by low insertion loss and a high $Q$ [3]. However, a major drawback of silicon resonators is in their high TCF, which is in the range of -20 to -30 $\mathrm{ppm} /{ }^{\circ} \mathrm{C}$ for uncompensated resonators [1] [2]. In order to compensate for this large negative TCF, a layer of a material with positive temperature coefficient of elasticity (typically $\mathrm{SiO}_{2}$ ) can be added to the resonator stack [1-3]. However, in order to achieve full temperature compensation in length-extensional resonators, the thicknesses of the $\mathrm{SiO}_{2}$ and $\mathrm{Si}$ layers should be comparable. This creates several problems, given that a relatively thick layer of silicon $(20-60 \mu \mathrm{m})$ is required in AlN-transduced resonators to achieve a high $Q$ as well as good power handling and linearity. One problem is that the addition of a comparably thick $\mathrm{SiO}_{2}$ layer will result in a considerable drop in the device $Q$. Secondly, a thick $\mathrm{SiO}_{2}$ layer makes the fabrication process challenging. In order to address these challenges, we present a new method of passive material compensation. Instead of adding a thick layer of oxide, a uniform array of $\mathrm{SiO}_{2}$ pillars are formed in the silicon body of the resonator to generate a composite structure with a near zero TCF. The pillars are formed by filling square-shaped trenches etched in the body of the resonator with high-temperature $\mathrm{LPCVD}^{\mathrm{SiO}}{ }_{2}$. In contrast to oxide-compensated capacitive resonators, the presented resonators do not require a DC electric field for operation and are hence immune from dielectric charging effects that cause frequency drift [4].

\section{RESONATOR DESIGN}

A silicon resonator with a two-dimensional array of uniformly distributed oxide pillars can be modeled as a network of series and parallel finite-mass springs. Figure 1 shows a typical array of oxide pillars and its equivalent mass-spring network model.

The equivalent mass and stiffness of the springs, which determine the resonance frequency of the system and its variation with temperature, depend on the distribution and configuration of the oxide pillars. Assuming a totally uniform pattern, the overall amount of oxide required to fully compensate the negative TCF of the silicon resonator can be estimated from the following equation:

$$
\frac{1}{2}\left(\frac{\rho_{o x}^{1 / 2} E_{s i}^{1 / 2}}{\rho_{s i}^{1 / 2} E_{o x}^{1 / 2}}+\frac{E_{o x}}{E_{s i}}\right)\left(\frac{T C E_{o x}}{T C E_{s i}}\right)\left(\frac{V_{o x}}{V_{s i}}\right)=-1
$$

Here $V_{o x}$ and $V_{s i}$ are the overall volume of oxide pillars and silicon in the resonator, $E_{o x}$ and $E_{s i}$ are Young's Modulus and $\rho_{o x}$ and $\rho_{s i}$ are density of oxide and silicon respectively.

ANSYS simulations were used to adjust the dimensions and distribution of the oxide pillars and the thickness of the top oxide layer in order to achieve zero TCF over the desired range of temperature at a specific resonance frequency.

\section{RESONATOR FABRICATION}

To fabricate the resonators, trenches with slightly tapered sidewalls are first etched into the device layer of an SOI wafer and subsequently filled with $\mathrm{LPCVD} \mathrm{SiO}_{2}$ deposited at $850^{\circ} \mathrm{C}$. A smooth top surface is obtained by short wet etching and subsequent deposition of $\mathrm{SiO}_{2}$. A nearly smooth surface is required for the deposition of Mo electrodes and high-quality AlN. The rest of the fabrication process is similar to the one reported in [3] with some minor modifications. Figure 2 shows an SEM picture of a fabricated temperature-stable $24 \mathrm{MHz}$ resonator with uniformly distributed array of oxide pillars.

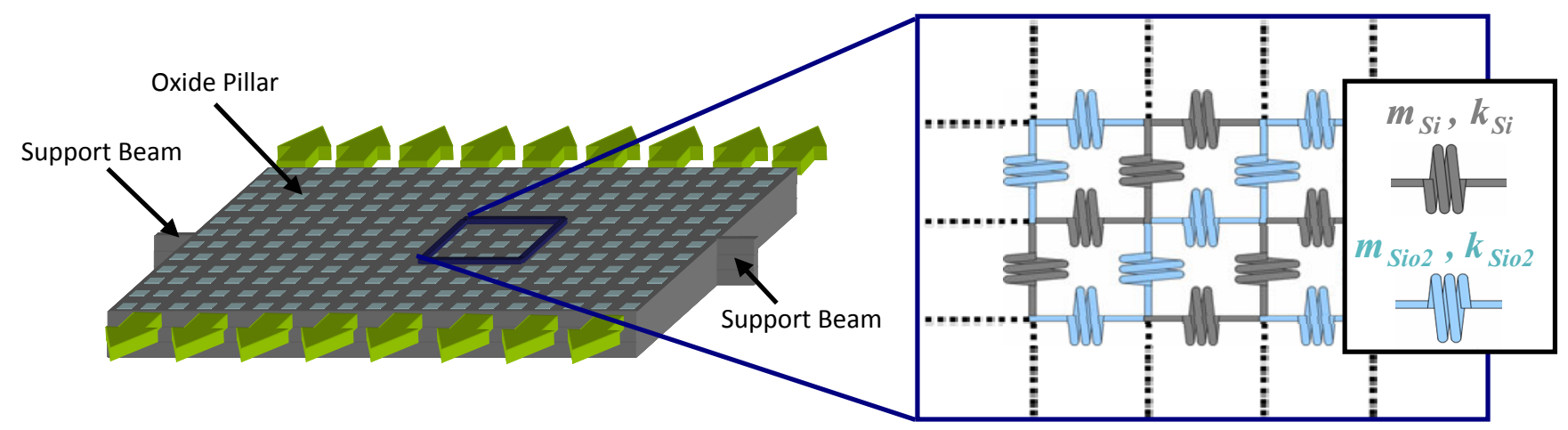

Figure 1: A typical array of oxide pillars in resonator and simplified equivalent mass-spring model. Arrows show resonance direction. 


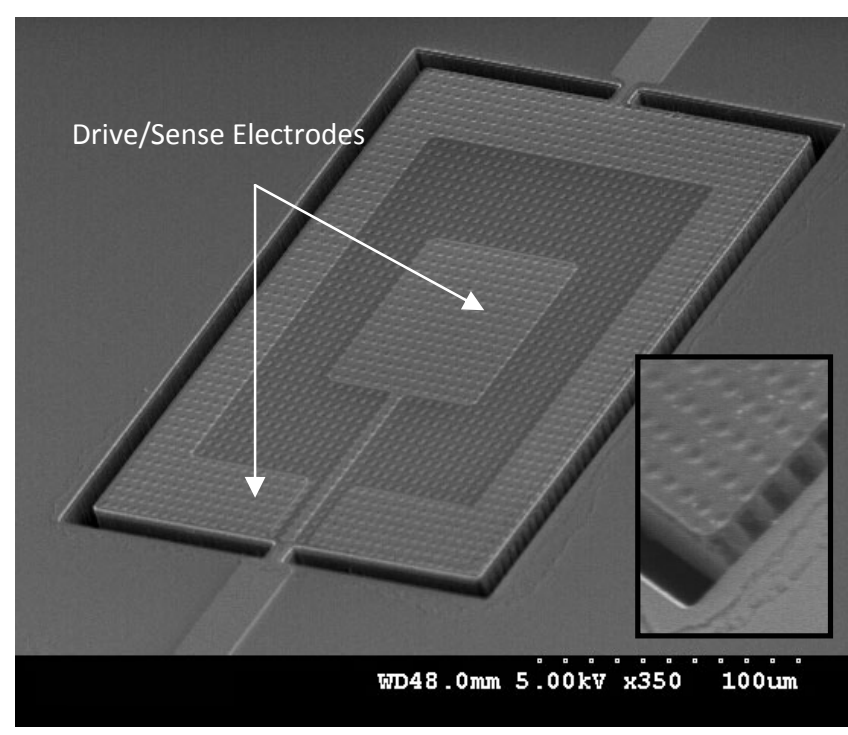

Figure 2: SEM picture of a temperature compensated $24 \mathrm{MHz}$ resonator with $\mathrm{SiO}_{2}$ pillars. Inset shows the oxide pillars.

\section{CHARACTERIZATION RESULTS}

Figure 3 is the measured frequency response of the temperature stable resonator of Figure 2 showing very high $Q$.

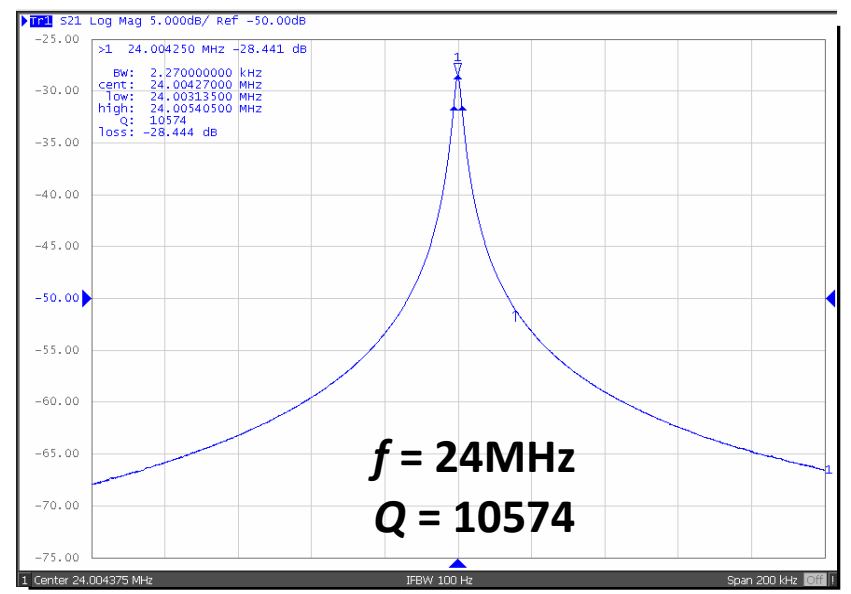

Figure 3: Frequency response of the $24 \mathrm{MHz}$ temperature compensated resonator of Figure 2.

Figure 4 shows the temperature characteristics of three identical samples of compensated $24 \mathrm{MHz}$ resonators from across the wafer with a $1.4 \mu \mathrm{m}$ of surface LPCVD oxide layer deposited between a $20 \mu \mathrm{m}$ layer of silicon body and Mo/AlN/Mo layers. A resonance frequency drift of $90-180 \mathrm{ppm}$ over the range $-20{ }^{\circ} \mathrm{C}$ to $100{ }^{\circ} \mathrm{C}$ was measured for these three samples while $Q$ exceeded 10,000 at all temperatures (in vacuum). Figure 5 shows the temperature characteristic of $Q$ for the resonator of Figure 2.

\section{ACKNOWLEDGMENT}

This work has been supported by Integrated Device Technology, Inc.

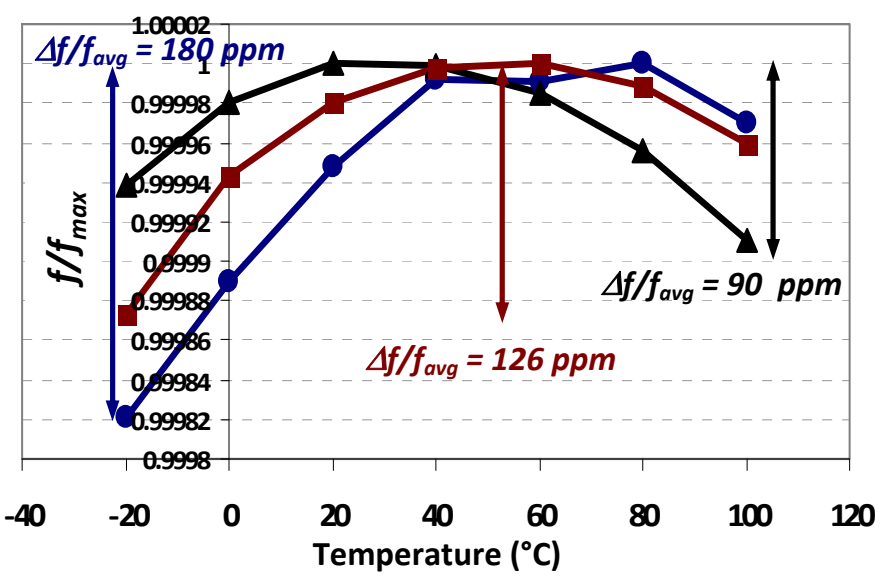

Figure 4: Temperature characteristic of three $24 \mathrm{MHz}$ temperature compensated AlN-on-Si resonators with oxide pillars.

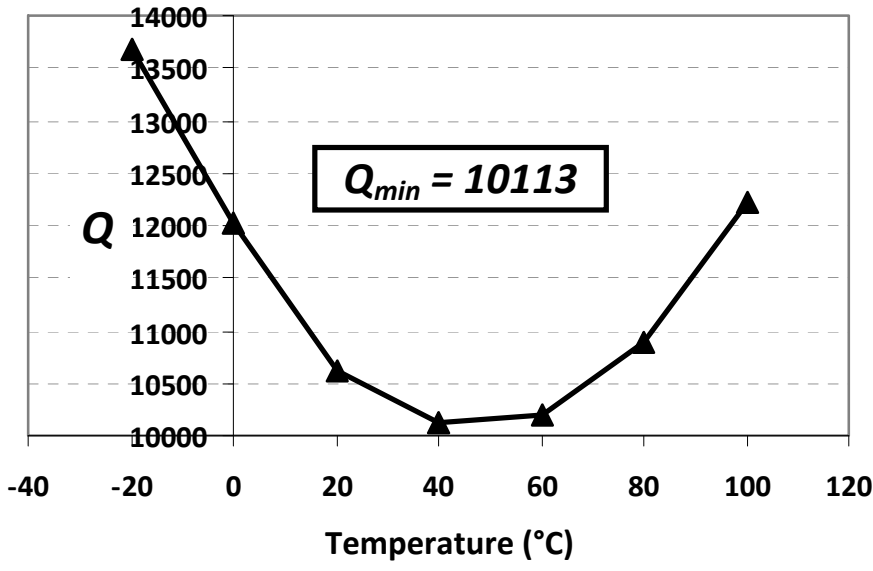

Figure 5: Temperature characteristic of $Q$ for the resonator of Figure 2.

\section{REFERENCES}

[1] F. Ayazi, "MEMS for Integrated Timing and Spectral Processing," Invited Paper, Proc. IEEE Custom Integrated Circuits Conference (CICC 2009), Sept. 2009, pp. 65-72

[2] R. Abdolvand, H. Miri Lavasani, and F. Ayazi, "A Low Voltage Temperature-Stable Micromechanical Piezo Oscillator," Tech. Digest of the 14th International Conference on Solid State Sensors, Actuators and Microsystems (Transducers'07), Lyon, France, June 2007, pp. 53-56.

[3] W. Pan and F. Ayazi, "Thin-Film Piezoelectric-on-Substrate Resonators with Q Enhancement and TCF Reduction," Tech. Digest of 23rd IEEE International Conference on Micro Electro Mechanical Systems (MEMS 2010), Hong Kong, January 24 - 28, 2010, pp. 104-107

[4] G. Bahl et al, "Observation of fixed and mobile charge in composite MEMS resonators", Solid-State Sensors, Actuators, and Microsystems Workshop, Hilton Head 2008, Hilton Head, SC, USA, pp 102-105

\section{CONTACT}

R.Tabrizian, tel: +1-404-259-7322; Roozbeh@gatech.edu

F. Ayazi, ayazi@gatech.edu 